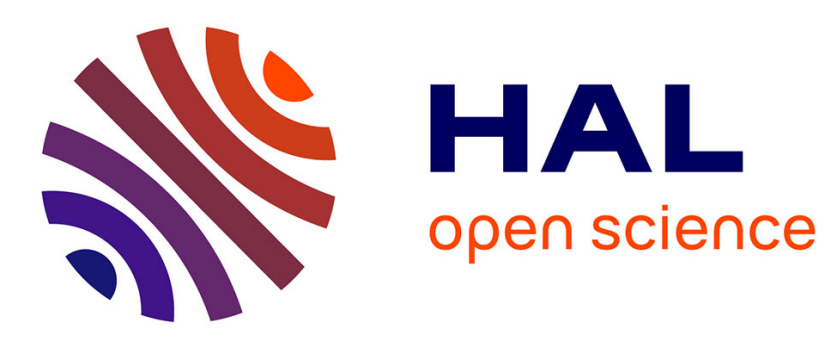

\title{
Asymptotic tracking of a state trajectory by output-feedback for a class of non linear systems
}

Vincent Andrieu, Laurent Praly, Alessandro Astolfi

\section{To cite this version:}

Vincent Andrieu, Laurent Praly, Alessandro Astolfi. Asymptotic tracking of a state trajectory by output-feedback for a class of non linear systems. Decision and Control, 2007 46th IEEE Conference on, Dec 2007, New Orleans, United States. pp.5228 - 5233, 10.1109/CDC.2007.4434422 . hal-00457835

\section{HAL Id: hal-00457835 \\ https://hal.science/hal-00457835}

Submitted on 18 Feb 2010

HAL is a multi-disciplinary open access archive for the deposit and dissemination of scientific research documents, whether they are published or not. The documents may come from teaching and research institutions in France or abroad, or from public or private research centers.
L'archive ouverte pluridisciplinaire HAL, est destinée au dépôt et à la diffusion de documents scientifiques de niveau recherche, publiés ou non, émanant des établissements d'enseignement et de recherche français ou étrangers, des laboratoires publics ou privés. 


\title{
Asymptotic tracking of a state trajectory by output-feedback for a class of non linear systems
}

\author{
V. Andrieu, L. Praly and A. Astolfi
}

\begin{abstract}
We consider the problem of tracking a reference trajectory with an output feedback for a class of nonlinear systems. We solve this problem by combining the techniques of dynamic scaling and homogeneity in the bi-limit.
\end{abstract}

\section{INTRODUCTION}

We address the following problem : Given a system $\dot{\eta}=$ $f(\eta, u)$ with output $y=h(\eta)$, and a bounded reference trajectory $\left(\eta_{r}, u_{r}\right)$, exact solution of $\dot{\eta}_{r}=f\left(\eta_{r}, u_{r}\right)$, design an output feedback $u=\varphi\left(w, y, \eta_{r}\right), \dot{w}=\theta\left(w, y, \eta_{r}\right)$ which ensures global convergence of $\eta$ to $\eta_{r}$.

To illustrate our contribution we consider the system $^{1}$ :

$$
\left\{\begin{array}{l}
\dot{z}=-z+x_{2} \\
\dot{x}_{1}=x_{2}, \\
\dot{x}_{2}=u+x_{2}^{1+d}+z, \\
y=x_{1} .
\end{array}\right.
$$

where $y$ in $\mathbb{R}$ is the available measurement, $u$ in $\mathbb{R}$ is the control input and $d$ a real number in $[-1,1)$. Consider a bounded reference trajectory $\left(z_{r}, x_{1 r}, x_{2 r}, u_{r}\right)$, exact solution of (1), namely :

$$
\left\{\begin{array}{l}
\dot{z}_{r}=-z_{r}+x_{2, r} \\
\dot{x}_{r, 1}=x_{r, 2} \\
\dot{x}_{r, 2}=u_{r}+x_{r, 2}^{1+d}+z_{r} .
\end{array}\right.
$$

The problem is to find an output feedback for $u$ such that $\left(z, x_{1}, x_{2}\right)$ converges to $\left(z_{r}, x_{r, 1}, x_{r, 2}\right)$. This can be rephrased as finding $\tilde{u}=u_{r}-u$, depending on $\left(z_{r}, \tilde{x}_{1}, x_{r, 1}, x_{r, 2}, u_{r}\right)$, rendering the origin of the error system :

$$
\left\{\begin{array}{l}
\dot{\tilde{z}}=-\tilde{z}+\tilde{x}_{2}, \\
\dot{\tilde{x}}_{1}=\tilde{x}_{2}, \\
\dot{\tilde{x}}_{2}=\tilde{u}+x_{r, 2}^{1+d}-\left(x_{r, 2}+\tilde{x}_{2}\right)^{1+d}+\tilde{z},
\end{array}\right.
$$

globally attractive.

To solve this problem we follow a domination approach based on homogeneity. This leads to regard the term $x_{r, 2}^{1+d}-$ $\left(x_{r, 2}+\tilde{x}_{2}\right)^{1+d}+\tilde{z}$ as a perturbation which can be upperbounded as :

$$
\begin{aligned}
\mid x_{r, 2}^{1+d}-\left(x_{r, 2}\right. & \left.+\tilde{x}_{2}\right)^{1+d}+\tilde{z} \mid \\
& \leq(1+d)\left|x_{r, 2}\right|^{d}\left|\tilde{x}_{2}\right|+\left|\tilde{x}_{2}\right|^{1+d}+|\tilde{z}|
\end{aligned}
$$

V. Andrieu is with the Electrical and Electronic Engineering Dept, Imperial College, London, SW7 2AZ, UK (vincent. andrieu@gmail.com)

L. Praly is with CAS - Ecole des Mines de Paris, 35, rue St-Honore, 77305 Fontainebleau CEDEX, France, (laurent.praly@ensmp. fr)

A. Astolfi is with the Electrical and Electronic Engineering Dept, Imperial College, London, SW7 2AZ, UK (a.astolfi@ic.ac.uk) and with Dipartimento di Informatica Sistemi e Produzione, University of Rome Tor Vergata, Via del Politecnico 1, 00133 Roma, Italy

${ }^{1}$ For any real numbers $w$ and $r, w^{r}$ denotes $\operatorname{sign}(w)|w|^{r}$.
The term $(1+d)\left|x_{r, 2}\right|^{d}$ is a known function which multiplies a linear function of the error. To deal with this kind of term, we follow an idea introduced in [14] and design a high-gain output feedback with a dynamic scaling with a gain updated from the reference signal $x_{r, 2}$.

The second term, namely $\left|\tilde{x}_{2}\right|^{1+d}$, is a power of the norm of the error $\left|\tilde{x}_{2}\right|$. To deal with this term, we use the homogeneous in the bi-limit output feedback design tool we have introduced in [1] and further developed in [2] (see the Appendix).

Finally, the term $|\tilde{z}|$ depends on the zero dynamics. We deal with this one by imposing a minimum phase assumption and invoking a small gain argument.

In conclusion, our solution to this tracking problem is based on a domination approach and combines high-gain with dynamic scaling and homogeneity in the bi-limit.

When compared with what can be found in the textbooks [13], [12] for instance, our approach allows us to deal with some polynomial terms in the unmeasured state components. This type of extension is already present in [8] where a practical tracking result is obtained.

In section II the main results of the paper are stated and commented. In Section III, we introduce an extension of the homogeneous in the bi-limit output feedback design for a chain of integrator introduced in [1] to render it compatible with the use of dynamic scaling. This tool is then exploited in Sections IV to introduce the dynamic high-gain outputfeedback.

\section{MAin RESUlt OF THE PAPER}

Consider a system whose dynamics are :

$$
\left\{\begin{aligned}
\dot{z} & =F(z, x), \\
\dot{x}_{1} & =x_{2}+\delta_{1}(z, x), \\
\dot{x}_{2} & =x_{3}+\delta_{2}(z, x), \quad y=x_{1} . \\
& \vdots \\
\dot{x}_{n} & =u+\delta_{n}(z, x),
\end{aligned}\right.
$$

where $x=\left(x_{1}, \ldots x_{n}\right)$ in $\mathbb{R}^{n}, y$ is the output in $\mathbb{R}, u$ is the input in $\mathbb{R}$ and $z$ in $\mathbb{R}^{n_{z}}$ is the state of the inverse dynamics. This state can be "neglected" provided the inverse dynamics with $\delta_{i}$ as output and $x$ as input are incremental ISS (see [3]). Specifically, we make the following assumption.

Assumption 1 : There exist a real number $d_{\infty}$ in $\left[0, \frac{1}{n-1}\right)$, positive real numbers $v, c_{\infty}, p$ and $q$, non negative $C^{1}$ functions $Z_{i}$, non negative continuous functions $\Omega, \gamma_{i}$ and $\mu_{i}$, a function $\alpha$ of class $\mathcal{K}_{\infty}$ and a continuous function $\omega$, with strictly positive values such that, for all $i$, 
$1.1 \mu_{i}^{p}$ is $C^{1}$, convex and satisfies $s \mu_{i}^{p \prime}(s) \leq q \mu_{i}^{p}(s)$, $\mu_{i}(0)=0$,

$1.2 \alpha(|\tilde{z}|) \omega(|z|) \leq \sum_{i=1}^{n} \mu_{i}\left(Z_{i}(z, \tilde{z})\right)$,

$1.3 \frac{\partial Z_{i}}{\partial z}(z, \tilde{z}) F(z, x)$

$$
\begin{array}{r}
+\frac{\partial Z_{i}}{\partial \tilde{z}}(z, \tilde{z})[F(z+\tilde{z}, x+\tilde{x})-F(z, x)] \\
\leq-Z_{i}(z, \tilde{z})+\gamma_{i}(\tilde{x}),
\end{array}
$$

$1.4\left|\delta_{i}(z+\tilde{z}, x+\tilde{x})_{i}-\delta_{i}(z, x)\right|$

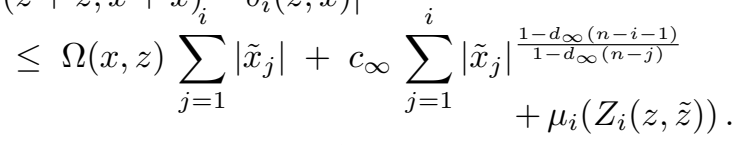

$1.5 \mu_{i}\left((1+v) \gamma_{i}(\tilde{x})\right) \leq c_{\infty} \sum_{j=1}^{i}\left|\tilde{x}_{j}\right|+\left|\tilde{x}_{j}\right|^{\frac{1-d_{\infty}(n-i-1)}{1-d_{\infty}(n-j)}}$.

In Section IV, we prove :

Theorem 1 (Main result): Consider system (4) with Assumption 1. Then the controller given in (16) solves the tracking problem for any bounded reference trajectory $t \mapsto$ $\left(z_{r}(t), x_{r}(t), u_{r}(t)\right)$ which is a particular solution of (4).

For the system (1), the condition 1.4 of Assumption 1 follows from inequality (3) by setting $d_{\infty}=d$ and picking $\Omega\left(x_{r}, z_{r}\right)=(1+d)\left|x_{r, 2}\right|^{d}, Z_{2}\left(z, z_{r}\right)=|\tilde{z}|^{2}, \mu_{2}(s)=\sqrt{s}$, $\gamma_{2}(\tilde{x})=\left|\tilde{x}_{2}\right|^{2}$. Consequently, system (1) belongs to the class of systems satisfying Assumption 1 and Theorem 1 applies.

Assumption 1 is to be compared with the one in [8] where standard homogeneity and domination is also used. In that contribution, there is no $z$ dynamics and the objective is only practical tracking. This allows the authors to work with an assumption weaker than 1.4 since it is needed only for $(x, z, \tilde{x}, \tilde{z})=(0,0, \tilde{x}, \tilde{z})$. (See footnote 4). Here we get exact tracking but under the more restrictive assumption that the reference trajectory is an exact solution of the system to be controlled. [4] and [11] are two other contributions where such a more restrictive assumptions is not needed, but on the other hand they do not allow polynomial growth in the $\delta_{i}$.

Another important point is that we do not need to know in advance the whole reference trajectory or even a bound on it to design the controller. This is to be opposed for instance to the controller proposed in [8]. Compare also with [11].

\section{HOMOGENEOUS TOOLS FOR A CHAIN OF INTEGRATOR}

Throughout this section we consider a chain of integrator, with state $\mathfrak{X}=\left(\mathcal{X}_{1}, \ldots, \mathcal{X}_{n}\right)$ in $\mathbb{R}^{n}$ described by :

$$
\dot{\mathfrak{X}}=\mathcal{S} \mathfrak{X}+B u \quad, \quad y=\mathcal{X}_{1},
$$

where $B=(0, \ldots, 1)^{T}$ and $\mathcal{S}$ denotes the left shift matrix of order $n$, i.e.

$$
\mathcal{S} \mathfrak{X}=\left(\mathcal{X}_{2}, \ldots, \mathcal{X}_{n}, 0\right)^{T} .
$$

We deal now with homogeneity in the bi-limit. This notion and its properties are studied in [2]. We give in the Appendix a brief summary.
Selecting arbitrary degrees $d_{0} \leq d_{\infty}$ in $\left(-1, \frac{1}{n-1}\right)$, homogeneity in the bi-limit is obtained for system (5) provided the weights $r_{0}=\left(r_{0,1}, \ldots, r_{0, n}\right)$ and $r_{\infty}=\left(r_{\infty, 1}, \ldots, r_{\infty, n}\right)$ are :

$$
r_{0, i}=1-d_{0}(n-i), \quad r_{\infty, i}=1-d_{\infty}(n-i) .
$$

\section{A. Homogeneous in the bi-limit observer}

In [1], [2], we have proposed an observer for system (5) given by :

$$
\dot{\hat{\mathfrak{X}}}=\mathcal{S} \hat{\mathfrak{X}}+B u+K\left(\hat{\mathcal{X}}_{1}-\mathcal{X}_{1}\right),
$$

where $\hat{\mathfrak{X}}=\left(\hat{\mathcal{X}}_{1}, \ldots, \hat{\mathcal{X}}_{n}\right)$ in $\mathbb{R}^{n}$, and $K$ is a homogeneous in the bi-limit vector field with weights $r_{0}$ and $r_{\infty}$, and degrees $d_{0}$ and $d_{\infty}$. Setting :

$$
E=\left(e_{1} \ldots, e_{n}\right)^{T}=\hat{\mathfrak{X}}-\mathfrak{X}
$$

yields the error system :

$$
\dot{E}=S E+K\left(e_{1}\right) .
$$

The design of $K$ is done recursively in such a way that there exists a homogeneous in the bi-limit Lyapunov function $W$ of degree $d_{W}$ satisfying, for some real number $c_{1}$,

$$
\begin{aligned}
\frac{\partial W}{\partial E}(E)(\mathcal{S} & \left.E+K\left(e_{1}\right)\right) \\
\leq & -c_{1}\left(W(E)^{\frac{d_{W}+d_{0}}{d_{W}}}+W(E)^{\frac{d_{W}+d_{\infty}}{d_{W}}}\right),
\end{aligned}
$$

To combine this tool with dynamic scaling we need to establish a specific property on the error Lyapunov function $W$. This property is a homogeneous in the bi-limit version of the one given in [14, equation (16)] or in [9, Lemma A1]. Namely, given the diagonal matrix

$$
D=\operatorname{diag}\left(d_{1}, \ldots, d_{n}\right),
$$

with $d_{i}>0$, the function $K$ has to be selected such that the associated error Lyapunov function $W$ satisfies (9), and also :

$$
\frac{\partial W}{\partial E}(E) D E \geq c_{2} W(E)
$$

for some positive real number $c_{2}$.

Such a property can be obtained by modifying ${ }^{2}$ the recursive procedure given in [1], [2] as claimed in the following statement the proof of which is omitted.

Theorem 2: Let $d_{W}$ be a positive real number satisfying $d_{W} \geq 2 \max _{1 \leq j \leq n} r_{0, j}+d_{\infty}$ and $D=\operatorname{diag}\left(d_{1}, \ldots, d_{n}\right)$ with $d_{j}>0$. There exists a homogeneous in the bi-limit vector field $K: \mathbb{R} \rightarrow \mathbb{R}^{n}$, with associated triples $\left(r_{0}, d_{0}, K_{0}\right)$ and $\left(r_{\infty}, d_{\infty}, K_{\infty}\right)$ and a positive definite, proper and $C^{1}$ function $W: \mathbb{R}^{n} \rightarrow \mathbb{R}_{+}$, homogeneous in the bi-limit with associated triples $\left(r_{0}, d_{W}, W_{0}\right)$ and $\left(r_{\infty}, d_{W}, W_{\infty}\right)$, such that :

1) The functions $W_{0}$ and $W_{\infty}$ are positive definite and proper and the functions $\frac{\partial W}{\partial e_{j}}$ are homogeneous in the bi-limit

\footnotetext{
${ }^{2}$ We multiply $W_{i+1}$ by a sufficiently large number before using it in the definition of $W_{i}$
} 
2) There exist two positive real numbers $c_{1}$ and $c_{2}$ such that (9) and (10) are satisfied.

\section{B. Homogeneous in the bi-limit state feedback}

In the same spirit as the homogeneous in the bi-limit observer design introduced in the previous section, we modify ${ }^{3}$ the recursive state feedback design introduced in [1] to make it compatible with dynamic scaling. The result is expressed in the following statement.

Theorem 3: Let $d_{V}$ be a positive real number satisfying $d_{V}>2 \max 1 \leq j \leq n r_{0, j}$, and $D=\left(d_{1}, \ldots, d_{n}\right)$, with $d_{j}>0$. There exist a homogeneous in the bi-limit function $\phi: \mathbb{R}^{n} \rightarrow \mathbb{R}$, with associated triples $\left(r_{0}, 1+d_{0}, \phi_{0}\right)$ and $\left(r_{\infty}, 1+d_{\infty}, \phi_{\infty}\right)$ and a positive definite, proper and $C^{1}$ function $V: \mathbb{R}^{n} \rightarrow \mathbb{R}_{+}$homogeneous in the bi-limit with associated triples $\left(r_{0}, d_{V}, V_{0}\right)$ and $\left(r_{\infty}, d_{V}, V_{\infty}\right)$, such that :

1) The functions $V_{0}$ and $V_{\infty}$ are positive definite and proper and the functions $\frac{\partial V}{\partial \mathcal{X}_{j}}$ are homogeneous in the bi-limit

2) There exists $c_{3}>0$ such that, for all $\mathfrak{X}$ in $\mathbb{R}^{n}$,

$$
\begin{aligned}
\frac{\partial V}{\partial \mathfrak{X}}(\mathfrak{X})(\mathcal{S} \mathfrak{X} & +B \phi(\mathfrak{X})) \\
& \leq-c_{3}\left(V(\mathfrak{X})^{\frac{d_{V}+d_{0}}{d_{V}}}+V(\mathfrak{X})^{\frac{d_{V}+d_{\infty}}{d_{V}}}\right) .
\end{aligned}
$$

3) There exists $c_{4}>0$ such that, for all $\mathfrak{X}$ in $\mathbb{R}^{n}$,

$$
\frac{\partial V}{\partial \mathfrak{X}}(\mathfrak{X}) D \mathfrak{X} \geq c_{4} V(\mathfrak{X}) .
$$

\section{PROOF OF THEOREM 1}

\section{A. Rephrasing the problem as a stabilization problem}

Setting

$$
\tilde{u}=u-u_{r}, \quad \tilde{x}=x-x_{r}, \quad \tilde{z}=z-z_{r},
$$

we obtain :

$$
\left\{\begin{array}{c}
\dot{\tilde{z}}=F\left(z_{r}+\tilde{z}, x_{r}+\tilde{x}\right)-F\left(z_{r}, x_{r}\right), \\
\dot{\tilde{x}}_{1}=\tilde{x}_{2}+\delta_{1}\left(z_{r}+\tilde{z}, x_{r}+\tilde{x}\right)-\delta_{1}\left(z_{r}, x_{r}\right), \\
\vdots \\
\dot{\tilde{x}}_{n}=\tilde{u}+\delta_{n}\left(z_{r}+\tilde{z}, x_{r}+\tilde{x}\right)-\delta_{1}\left(z_{r}, x_{r}\right) .
\end{array}\right.
$$

The objective is now to find $\tilde{u}$ depending on the output $\tilde{x}_{1}=$ $y-x_{r, 1}$ and on the reference trajectory $\left(z_{r}, x_{r}\right)$ such that the solution $\tilde{x}=0$ is globally attractive ${ }^{4}$.

\section{B. Output feedback using homogeneous in the bi-limit tools and dynamic scaling}

The output feedback is obtained from the functions $K$ and $\phi$ given by Theorems 2 and 3 and by introducing an extra dynamically updated gain $L$.

The first step consists in selecting the parameter $D$ in Theorem 2 and 3. Following [14], let :

\footnotetext{
${ }^{3}$ We multiply $V_{i}$ by a sufficiently large number before using it in the definition of $V_{i+1}$

${ }^{4}$ In [8], $\tilde{x}$ is defined as $\tilde{x}=\left(\left(x_{1}-y_{r}, x_{2}, \ldots, x_{n}\right)\right.$. This leads to the presence of $\dot{y}_{r}$ as a disturbance in the $\tilde{x}$ dynamics explaining why practical tracking is obtained and why a weaker assumption can be invoked.
}

$$
D=\operatorname{diag}(b, 1+b, \ldots, n-1+b)^{T},
$$

where $b$ is a positive real number satisfying ${ }^{5}$,

$$
\frac{1-d_{\infty}(n-i-1)}{1-d_{\infty}(n-j)}<\frac{i+b}{j-1+b}<\frac{i}{j-1},
$$

for all $1 \leq j \leq i \leq n$ and with $d_{\infty}$ as given in Assumption 1 .

Selecting $d_{0}=0$ and $d_{W}=d_{V}=d_{U}$ sufficiently large, we apply Theorem 2 and 3 to construct the homogeneous in the bi-limit vector field $K$, the state feedback $\phi$ and the Lyapunov functions $W$ and $V$ such that (9), (10), (11) and (12) hold.

Following [1], the output feedback is given by :

$$
\begin{aligned}
& u=u_{r}+L^{n+b} \phi\left(\mathfrak{L}^{-1} \hat{x}\right), \\
& \dot{\hat{x}}=\mathcal{S} \hat{x}+B L^{n+b} \phi\left(\mathfrak{L}^{-1} \hat{x}\right)+L \mathfrak{L} K\left(\frac{\hat{x}_{1}-\left(y-x_{r, 1}\right)}{L^{b}}\right),
\end{aligned}
$$

with :

$$
\mathfrak{L}=\operatorname{diag}\left(L^{b}, \ldots, L^{n+b-1}\right)
$$

and $L$ satisfying :

$$
\begin{aligned}
& \dot{L}=-a_{1} a_{22} L \\
& \quad+L \max \left\{0, a_{1}\left(a_{21}-L\right)+a_{3} \Omega\left(z_{r}, x_{r}\right)\right\},
\end{aligned}
$$

where $a_{1}, a_{21}, a_{22}$ and $a_{3}$ are positive real numbers to be defined with $a_{2}=a_{21}-a_{22}>0$.

This update law of the gain $L$ is an elaboration from the original one introduced in [14, (24)] and modified in $[5,(3.12)]$ or $[10,(134)]$. Note that the driving term depends only on the reference trajectory $\left(x_{r}, z_{r}\right)$. Since this trajectory is bounded, $L$ is bounded along any closedloop solution. Also we see that, if initialized to a value larger than $a_{2}, L$ remains larger than $a_{2}$ along any closedloop solution. Moreover the presence of the term $-a_{1} a_{22} L$ allows to recover the main property of $[14,(24)]$, i.e. $L$ "follows" its driving term. Specifically the Dini derivative of $\left|L-\frac{a_{1} a_{2}+a_{3} \Omega\left(z_{r}, x_{r}\right)}{a_{1}}\right|$ satisfies

$$
\begin{aligned}
D^{+}\left|L-\frac{a_{1} a_{2}+a_{3} \Omega\left(z_{r}, x_{r}\right)}{a_{1}}\right| \leq \frac{a_{3}}{a_{1}}\left|\overparen{\Omega\left(z_{r}, x_{r}\right)}\right| \\
-a_{1} \min \left\{a_{22}, a_{2}\right\}\left|L-\frac{a_{1} a_{2}+a_{3} \Omega\left(z_{r}, x_{r}\right)}{a_{1}}\right| .
\end{aligned}
$$

Hence along the solution of the closed loop system, we have

$$
\begin{array}{r}
\limsup _{t \rightarrow+\infty}\left|L(t)-\frac{a_{1} a_{2}+a_{3} \Omega\left(z_{r}(t), x_{r}(t)\right)}{a_{1}}\right| \leq \\
\leq \frac{a_{3}}{\min \left\{a_{22}, a_{2}\right\}} \limsup _{t \rightarrow+\infty}\left|\overparen{\Omega\left(z_{r}(t), x_{r}(t)\right)}\right| .
\end{array}
$$

Properties of the closed loop system : Let

$$
E=\left(e_{1}, \ldots, e_{n}\right)^{T}, \quad \hat{\mathfrak{X}}=\left(\hat{\mathcal{X}}_{1}, \ldots, \hat{\mathcal{X}}_{n}\right)^{T}
$$

and $\tau$ be scaled quantities defined as :

${ }^{5}$ This choice is always possible since, for $1 \leq j \leq i \leq n$, we have :

$$
\frac{i+b}{j-1+b}<\frac{i}{j-1} \quad \forall b>0,
$$

and 


$$
\begin{aligned}
& \frac{d E}{d \tau}=\mathcal{S} E+K\left(e_{1}\right) \quad-L^{-1} \frac{d L}{d \tau} D E \quad-\mathfrak{D}(L), \\
& \underbrace{\frac{d \hat{\mathfrak{X}}}{d \tau}=\mathcal{S} \widehat{\mathfrak{X}}+B \phi(\hat{\mathfrak{X}})+K\left(e_{1}\right)}-\underbrace{L^{-1} \frac{d L}{d \tau} D \widehat{\mathfrak{X}}} \underbrace{\square} \\
& \text { Chain of integrator part Dynamic Scaling Nonlinearities }=\text { Disturbances }
\end{aligned}
$$

$$
E=\mathfrak{L}^{-1}(\hat{x}-\tilde{x}) \quad, \quad \hat{\mathfrak{X}}=\mathfrak{L}^{-1} \hat{x} \quad, \quad \frac{d}{d \tau}=\frac{1}{L} \frac{d}{d t} .
$$

The closed loop dynamics can be described by equation (17), the $z$ dynamics and equations ${ }^{6}$ (19) at the top of this page, where

$$
\mathfrak{D}(L)=L^{-1} \mathfrak{L}^{-1}\left(\delta\left(z_{r}+\tilde{z}, x_{r}+\tilde{x}\right)-\delta\left(z_{r}, x_{r}\right)\right)
$$

is regarded as a perturbation.

We consider now the homogeneous in the bi-limit Lyapunov function :

$$
U(\hat{\mathfrak{X}}, E)=V(\hat{\mathfrak{X}})+\ell W(E),
$$

where $\ell$ is a positive real number to be specified and $V$ and $W$ are given by Theorems 2 and 3 . Along the trajectories of system (19), the Lyapunov function satisfies in the time $\tau$ :

$$
\frac{d}{d \tau} U(\hat{\mathfrak{X}}, E) \leq T_{C I}+T_{D S}+T_{D i s t},
$$

with

$$
\begin{aligned}
& T_{C I}= \frac{\partial V}{\partial \hat{\mathfrak{X}}}(\hat{\mathfrak{X}})(\mathcal{S} \hat{\mathfrak{X}}+B \phi(\hat{\mathfrak{X}}) \\
&+\left.+K\left(e_{1}\right)\right) \\
&+\ell \frac{\partial W}{\partial E}(E)\left(\mathcal{S} E+K\left(e_{1}\right)\right), \\
& T_{D S}=-L^{-1} \frac{d L}{d \tau}\left(\frac{\partial V}{\partial \hat{\mathfrak{X}}}(\hat{\mathfrak{X}}) D \hat{\mathfrak{X}}+\ell \frac{\partial W}{\partial E}(E) D E\right), \\
& T_{D i s t}=-\ell \frac{\partial W}{\partial E}(E) \mathfrak{D}(L) .
\end{aligned}
$$

Bound on the term $T_{C I}$. Let $\eta$ and $\gamma$ be functions defined as :

$$
\begin{aligned}
\eta(\hat{\mathfrak{X}}, E) & =\frac{\partial V}{\partial \widehat{\mathfrak{X}}}(\widehat{\mathfrak{X}})\left(\mathcal{S} \hat{\mathfrak{X}}_{n}+B \phi_{n}\left(\hat{\mathfrak{X}}_{n}\right)+K\left(e_{1}\right)\right) \\
\gamma(E) & =\frac{\partial W}{\partial E}(E)\left(\mathcal{S} E+K\left(e_{1}\right)\right) .
\end{aligned}
$$

These two functions are continuous and homogeneous in the bi-limit with associated triples $\left(\left(r_{0}, r_{0}\right), d_{U}, \eta_{0}\right)$, $\left(\left(r_{\infty}, r_{\infty}\right), d_{U}+d_{\infty}, \eta_{\infty}\right) \quad$ and $\quad\left(\left(r_{0}, r_{0}\right), d_{U}, \gamma_{0}\right)$, $\left(\left(r_{\infty}, r_{\infty}\right), d_{U}+d_{\infty}, \gamma_{\infty}\right)$, where $\gamma_{0}, \gamma_{\infty}$ and $\eta_{0}, \eta_{\infty}$ are continuous functions. Furthermore, by $(9), \gamma(E)$ is non positive for all $E$ in $\mathbb{R}^{n}$ and by (11), we have, for all $(\hat{\mathfrak{X}}, E) \neq(0,0)$,

$$
\begin{array}{rll}
\gamma(E)=0 & \Rightarrow & \eta(\hat{\mathfrak{X}}, E)<0, \\
\gamma_{0}(E)=0 & \Rightarrow & \eta_{0}(\hat{\mathfrak{X}}, E)<0, \\
\gamma_{\infty}(E)=0 & \Rightarrow & \eta_{\infty}(\hat{\mathfrak{X}}, E)<0 .
\end{array}
$$

Consequently, by Claim A.2 in the Appendix, we can select $\ell$ such that $T_{C I}$ is negative definite and the same holds for the homogeneous approximation in the 0 -limit and in the $\infty$-limit. Then, Claim A.3, yields a positive real number $c_{5}$ such that :

$$
{ }^{6} \text { Note that } \overparen{\mathfrak{L}^{-1}}=-L^{-1} \dot{L} D \mathfrak{L}^{-1}
$$

$$
T_{C I} \leq-c_{5}\left(U(\hat{\mathfrak{X}}, E)+U(\hat{\mathfrak{X}}, E)^{\frac{d_{\infty}+d_{U}}{d_{U}}}\right)
$$

Note that $\ell$ and $c_{5}$ have been fixed.

Bound on the term $T_{D S}$. From Claim A.1, the function $(\hat{\mathfrak{X}}, E) \mapsto \frac{\partial V}{\partial \hat{\mathfrak{X}}}(\hat{\mathfrak{X}}) D \hat{\mathfrak{X}}+\ell \frac{\partial W}{\partial E}(E) D E$ is homogeneous in the bi-limit with associated weights $\left(r_{0}, r_{0}\right)$ and $\left(r_{\infty}, r_{\infty}\right)$ and degrees $d_{U}$ and $d_{U}$. Hence Claim A.3, as well as equations (10) and (12) yield positive real numbers $c_{6}$ and $c_{7}$ such that :

$$
c_{6} \geq \frac{\frac{\partial V}{\partial \hat{\mathfrak{X}}}(\hat{\mathfrak{X}}) D \hat{\mathfrak{X}}+\ell \frac{\partial W}{\partial E}(E) D E}{U(\hat{\mathfrak{X}}, E)} \geq c_{7} .
$$

Finally, using the expression of $\dot{L}$ in (17), this gives :

$T_{D S} \leq-c_{7} a_{3} \frac{\Omega\left(z_{r}, x_{r}\right)}{L} U(\hat{\mathfrak{X}}, E)+c_{6} a_{1} U(\hat{\mathfrak{X}}, E)$.

Bound on the term $T_{D i s t}$. Let

$$
\tilde{x}_{j}=L^{b+j-1}\left(\mathcal{X}_{j}-e_{j}\right),
$$

By Assumption 1.4 and equation (6), we have, for all $i$,

$\left|\mathfrak{D}_{i}(L)\right|$

$$
\begin{aligned}
& =L^{-i-b}\left|\delta_{i}\left(z_{r}+\tilde{z}, x_{r}+\tilde{x}\right)-\delta_{i}\left(z_{r}, x_{r}\right)\right|, \\
& \leq \Omega\left(z_{r}, x_{r}\right) \sum_{j=1}^{i} L^{j-1-i}\left|\hat{\mathcal{X}}_{j}-e_{j}\right| \\
& \quad+c_{\infty} L^{-i-b} \sum_{j=1}^{i}\left|L^{b+j-1}\left(\hat{\mathcal{X}}_{j}-e_{j}\right)\right|^{\frac{r_{\infty, i}+d_{\infty}}{r_{\infty, j}}} \\
&
\end{aligned}
$$

Inequalities (15) imply the existence of a real number $\epsilon>0$ such that

$$
L^{-\epsilon} \geq L^{(b+j-1) \frac{r_{\infty, i}+d_{\infty}}{r_{\infty, j}}-i-b} \quad \forall L \geq 1 .
$$

The condition $L \geq 1$ holds along all closed loop solutions if $a_{2} \geq 1$. Consequently, for all $(\hat{\mathfrak{X}}, E)$ in $\mathbb{R}^{2 n}$ and $L \geq a_{2} \geq 1$,

$$
\begin{aligned}
\left|\mathfrak{D}_{i}(L)\right| \leq & \frac{\Omega\left(z_{r}, x_{r}\right)}{L} \sum_{j=1}^{i}\left|\hat{\mathcal{X}}_{j}-e_{j}\right| \\
& +c_{\infty} a_{2}^{-\epsilon} \sum_{j=1}^{i}\left|\hat{\mathcal{X}}_{j}-e_{j}\right|^{\frac{r_{\infty, i}+d_{\infty}}{r_{\infty, j}}}+L^{-i-b} \mu_{i}\left(Z_{i}\right) .
\end{aligned}
$$

On the other hand, the function $\left|\frac{\partial W}{\partial e_{i}}(E)\right|\left|\hat{\mathcal{X}}_{j}-e_{j}\right|$ (respectively $\left|\frac{\partial W}{\partial e_{i}}(E)\right|\left|\hat{\mathcal{X}}_{j}-e_{j}\right|^{\frac{r_{\infty, i}+d_{\infty}}{r_{\infty}, j}}$ ) is homogeneous in the bi-limit with weights $\left(r_{0}, r_{0}\right)$ and $\left(r_{\infty}, r_{\infty}\right)$ and degrees $d_{U}$ and $d_{U}+r_{\infty, j}-r_{\infty, i}$ (respectively $d_{U}-1+\frac{r_{\infty, i}+d_{\infty}}{r_{\infty, j}}\left(\geq d_{U}\right)$ and $d_{\infty}+d_{U}$ ). Hence, Claim A.3 yields positive real numbers $c_{8}$ and $c_{9}$, such that : 


$$
\begin{aligned}
\frac{\left|T_{\text {Dist }}\right|}{\ell} \leq c_{8} \frac{\Omega\left(z_{r}, x_{r}\right)}{L} U(\hat{\mathfrak{X}}, E) & (24) \\
+c_{\infty} a_{2}^{-\epsilon} c_{9} & \left(U(\hat{\mathfrak{X}}, E)+U(\hat{\mathfrak{X}}, E)^{\frac{d_{U}+d_{\infty}}{d_{U}}}\right) \\
& +\sum_{i=1}^{n}\left|\frac{\partial W}{\partial e_{i}}(E)\right| L^{-i-b} \mu_{i}\left(Z_{i}\right) .
\end{aligned}
$$

Hence, by (22) and (23), selecting $a_{1}$ sufficiently small and $a_{2}$ and $a_{3}$ sufficiently large guarantees the existence of a positive real number $c_{10}$ such that (21) becomes :

$$
\begin{array}{r}
\frac{d U(\mathfrak{X}, E)}{d \tau} \leq-2 c_{10}\left(U(\hat{\mathfrak{X}}, E)+U(\hat{\mathfrak{X}}, E)^{\frac{d_{U}+d_{\infty}}{d_{U}}}\right) \\
+\sum_{i=1}^{n}\left|\frac{\partial W}{\partial e_{i}}(E)\right| L^{-i-b} \mu_{i}\left(Z_{i}\right) .
\end{array}
$$

Small-gain arguments. Let $\zeta_{i}$ and $\theta$ be the homogeneous in the bi-limit functions defined as

$\zeta_{i}(s)=|s|^{d_{U}}+|s|^{\frac{d_{U}+d_{\infty}}{r_{\infty}+d_{\infty}}}, \theta(s)=|s|+|s|^{\frac{d_{U}+d_{\infty}}{d_{U}}}$.

We use Claim A.2 with the functions

$-c_{10} \theta(U(\hat{\mathfrak{X}}, E))+\sum_{i=1}^{n}\left|\frac{\partial W}{\partial e_{i}}(E)\right| s_{i} \quad$ and $\quad \sum_{i=1}^{n} \zeta_{i}\left(s_{i}\right)$,

which are homogeneous in the bi-limit with weights $\left(r_{\infty}, r_{\infty}\right)$ and $\left(r_{0}, r_{0}\right)$ for $(\hat{\mathfrak{X}}, E)$, and $\left(1, r_{\infty, i}+d_{\infty}\right)$ for $s_{i}$ and degrees $d_{U}$ and $d_{\infty}+d_{U}$. This gives a positive real number $c_{11}$ satisfying :

$$
-c_{10} \theta(U(\hat{\mathfrak{X}}, E))+\sum_{i=1}^{n}\left|\frac{\partial W}{\partial e_{i}}(E)\right| s_{i} \leq c_{11} \sum_{i=1}^{n} \zeta_{i}\left(s_{i}\right) .
$$

Thus, inequality (25) (in time $t$ ) implies :

$$
\begin{aligned}
\dot{U}(\mathfrak{X}, E) \leq-c_{10} L & \theta(U(\hat{\mathfrak{X}}, E)) \\
& +c_{11} L \sum_{i=1}^{n} \zeta_{i}\left(L^{-i-b} \mu_{i}\left(Z_{i}\left(z_{r}, \tilde{z}\right)\right)\right) .
\end{aligned}
$$

Since, along solutions, $L$ is lower bounded by $a_{2}$, this implies the existence of a class $\mathcal{K} \mathcal{L}$ function $\beta_{u}$ such that we have, for all $t$ in the domain of existence of the closed loop solution and for all $s \leq t$

$$
\begin{aligned}
U(t) \leq \beta_{u}(U(s), t-s) & \\
& +\sup _{r \in[s, t)} \sum_{i=1}^{n} \theta^{-1}\left(\frac{2 c_{11}}{c_{10}} \zeta_{i}\left(L(r)^{-i-b} \mu_{i}\left(Z_{i}(r)\right)\right)\right),
\end{aligned}
$$

where to simplify the notation, we have defined

$$
U(t)=U(\mathfrak{X}(t), E(t)), Z_{i}(t)=Z_{i}\left(z_{r}(t), \tilde{z}(t)\right)
$$

and we have used the property $\theta(a)+\theta(b) \leq \theta(a+b)$.

Now, with $p$ as in Assumption 1, let

$$
\left.Y_{i}=L^{-p(i+b)} \mu_{i}\left(Z_{i}\left(z_{r}, \tilde{z}\right)\right)\right)^{p} .
$$

We get :

$$
\begin{aligned}
\dot{Y}_{i} \leq-p & (i+b) \frac{\dot{L}}{L} Y_{i} \\
& \left.\left.+L^{-p(i+b)} \mu_{i}^{p \prime}\left(Z_{i}\left(z_{r}, \tilde{z}\right)\right)\right)\left[-Z_{i}\left(z_{r}, \tilde{z}\right)\right)+\gamma_{i}(\tilde{x})\right] .
\end{aligned}
$$

Since $\dot{L}+a_{1} a_{22} L$ is non-negative, using property 1.1 of $\mu_{i}$ and considering the two cases $\left.(1+v) \gamma_{i}(\tilde{x}) \leq Z_{i}\left(z_{r}, \tilde{z}\right)\right)$ and $\left.Z_{i}\left(z_{r}, \tilde{z}\right)\right) \leq(1+v) \gamma_{i}(\tilde{x})$, we obtain

$$
\begin{aligned}
\dot{Y}_{i} \leq-\left[\frac{v}{1+v}-p(i+b) a_{1} a_{22}\right] & Y_{i} \\
& +q L^{-p(i+b)} \mu_{i}\left((1+v) \gamma_{i}(\tilde{x})\right)^{p} .
\end{aligned}
$$

We remark that the functions $\zeta_{i}^{-1}\left(\frac{c_{10}}{2 c_{11}} \theta(U(\hat{\mathfrak{X}}, E))\right)$ and $\left|\mathcal{X}_{j}-e_{j}\right|+\left|\mathcal{X}_{j}-e_{j}\right|^{\frac{r_{\infty, i}+d_{\infty}}{r_{\infty, j}}}$ are homogeneous in the bi-limit with weights $\left(r_{0}, r_{0}\right),\left(r_{\infty}, r_{\infty}\right)$ and degree 1 and $r_{\infty, i}+d_{\infty}$. Hence, using Claim A.3, we obtain the existence of a positive number $c_{12}$ such that :

$$
\begin{aligned}
c_{\infty} \sum_{j=1}^{i}\left|\mathcal{X}_{j}-e_{j}\right|+\mid \mathcal{X}_{j}- & \left.e_{j}\right|^{\frac{r_{\infty, i}+d_{\infty}}{r_{\infty, j}}} \\
& \leq c_{12} \zeta_{i}^{-1}\left(\frac{c_{10}}{2 c_{11}} \theta(U(\hat{\mathfrak{X}}, E))\right) .
\end{aligned}
$$

Moreover, since $L \geq a_{2} \geq 1$,

$$
\begin{aligned}
L^{-(i+b)} \sum_{j=1}^{i}\left|\tilde{x}_{j}\right|+ & \left|\tilde{x}_{j}\right|^{\frac{r_{\infty, i}+d_{\infty}}{r_{\infty}, j}} \\
& \leq a_{2}^{-\epsilon} \sum_{j=1}^{i}\left|\mathcal{X}_{j}-e_{j}\right|+\left|\mathcal{X}_{j}-e_{j}\right|^{\frac{r_{\infty, i}+d_{\infty}}{r_{\infty, j}}} .
\end{aligned}
$$

By Assumption 1.5, it follows that :

$$
\begin{aligned}
& \dot{Y}_{i} \leq-\left[\frac{v}{1+v}-p\right.\left.(i+b) a_{1} a_{22}\right] Y_{i} \\
&+q\left[a_{2}^{-\epsilon} c_{12} \zeta_{i}^{-1}\left(\frac{c_{10}}{2 c_{11}} \theta(U(\hat{\mathfrak{X}}, E))\right)\right]^{p} .
\end{aligned}
$$

Hence by selecting $a_{1}$ and $a_{22}$ to satisfy

$$
a_{1} a_{22}<\frac{v}{p(1+v)(n+b)},
$$

we obtain the existence of a class $\mathcal{K} \mathcal{L}$ function $\beta_{z}$ and a real number $c_{13}$ such that, for all $i$, all $t$ in the domain of existence of the closed loop solution and all $s \leq t$,

$$
\begin{aligned}
& \left.L(t)^{-(i+b)} \mu_{i}\left(Z_{i}(t)\right)\right) \\
& \leq \beta_{z}\left(L(s)^{-(i+b)} \mu_{i}\left(Z_{i}(s)\right), t-s\right) \\
& \quad+\sup _{r \in[s, t)} a_{2}^{-\epsilon} c_{13} \zeta_{i}^{-1}\left(\frac{c_{10}}{2 c_{11}} \theta(U(r))\right) .
\end{aligned}
$$

Since, by choosing $a_{2}$ large enough the small gain condition [6, condition (14)] is satisfied (see also [7]), we conclude there exists a function $\bar{\beta}_{u}$ of class $\mathcal{K} \mathcal{L}$ such that, on the domain of existence of the closed loop solution, and for all $i$,

$$
U(t) \leq \bar{\beta}_{u}\left(U(0)+\sum_{i=1}^{n} L(0)^{-(i+b)} \mu_{i}\left(Z_{i}(0)\right), t\right) .
$$

By Assumptions 1.2 and 1.3, there exists another function $\bar{\beta}_{z}$ of class $\mathcal{K} \mathcal{L}$ which depends on the bounds $\sup _{t} L(t)$ and $\sup _{t}\left|z_{r}(t)\right|$ such that, on the domain of existence of the closed loop solution,

$$
\alpha(|\tilde{z}(t)|) \leq \bar{\beta}_{z}\left(U(0)+\sum_{i=1}^{n} \mu_{i}\left(Z_{i}(0)\right), t\right) .
$$

This implies that the domain of existence is $[0,+\infty)$, and the global attractiveness of $\tilde{x}=E=0$ and $\tilde{z}=0$.

\section{CONClusion}

We have solved a state trajectory tracking problem for minimum phase non linear systems which admit globally a strict normal form and in such a way that the nonlinearities 
satisfy power growth. This has been achieved by exploiting the tools of domination, homogeneity in the bi-limit and dynamic scaling gain.

\section{REFERENCES}

[1] V. Andrieu, L. Praly, A. Astolfi Nonlinear Output Feedback Design Via Domination and Generalized Weighted Homogeneity. Proc. 45th IEEE CDC, San Diego, 2006.

[2] V. Andrieu, L. Praly, A. Astolfi, Homogeneous approximation, Recursive observer design and Output feedback. Submitted to SIAM, SICON

[3] D. Angeli, A Lyapunov approach to incremental stability. IEEE Transactions on Automatic Control, 47, pp. 410-422, 2002.

[4] A. Astolfi, G. Kaliora, Z.P. Jiang, Output feedback stabilization and approximate and restricted tracking for a class of cascaded systems IEEE Transactions on Automatic Control, 50, pp. 1390- 1396, 2005.

[5] Z. Chen, J. Huang Global robust servomechanism problem for uncertain lower triangular nonlinear systems by output feedback control. Proc. of the 43rd IEEE CDC, Bahamas, 2004.

[6] S. Dashkovskiy, B.S. Rüffer, F. Wirth, A small-gain type stability criterion for large scale networks of ISS systems, Proc. of the 44th EEE CDC, Sevilla, 2005.

[7] S. Dashkovskiy, B.S. Rüffer, F. Wirth, An ISS small gain theorem for general network, To appear in Mathematical Control, Signals and Systems.

[8] Q. Gong, C. Qian, Global practical tracking of a class of nonlinear systems by output feedback. Automatica, Vol. 43, pp. 184-189, (2007).

[9] P. Krishnamurthy, F. Khorrami, and R. S. Chandra, Global high-gain based observer and backstepping controller for generalized outputfeedback canonical form, IEEE Transactions on Automatic Control, vol. 48, No. 12, pp. 2277-2284, Dec. (2003).

[10] P. Krishnamurthy, F. Khorrami, Dynamic high-gain scaling : state and output feedback with application to systems with ISS appended dynamics driven by all states. IEEE Transactions on Automatic Control Vol. 49, No. 12, December 2004.

[11] P. Krishnamurthy, F. Khorrami and Z. P. Jiang, Global output feedback tracking for nonlinear systems in generalized output-feedback canonical form. IEEE Transactions on Automatic Control, Vol.47, no.5, pp.814-819, May 2002

[12] M. Krstić, I. Kanellakopoulos, P. Kokotović, Nonlinear and adaptive control design. John Wiley \& Sons, New York, (1995).

[13] R. Marino, P. Tomei, Nonlinear control design. Geometric, adaptive, robust, Prentice Hall, (1995).

[14] L. Praly, Asymptotic stabilization via output feedback for lower triangular systems with output dependent incremental rate. IEEE Trans. on Automat. Contr., Vol. 48, N. 6, June 2003.

[15] L. Praly, Z-P Jiang, Further Results on Robust Semiglobal Stabilization with Dynamic Input Uncertainties, Proc. of the 37th IEEE CDC, Tampa, 1998.

\section{APPENDIX}

\section{ON HOMOGENEITY IN THE BI-LIMIT}

For details on the notion of homogeneity in the bi-limit refer to [2]. We only give the definition and state the main properties used in this paper.

Given a vector $r=\left(r_{1}, \ldots, r_{n}\right)$ in $\left(\mathbb{R}_{+} /\{0\}\right)^{n}$, we define the dilation of a vector $x$ in $\mathbb{R}^{n}$ as

$$
\lambda^{r} \diamond x=\left(\lambda^{r_{1}} x_{1}, \ldots, \lambda^{r_{n}} x_{n}\right)^{T} .
$$

Definition 1 (Homogeneity in the 0-limit):

- A continuous function $\phi: \mathbb{R}^{n} \rightarrow \mathbb{R}$ is said homogeneous in the 0-limit with associated triple $\left(r_{0}, d_{0}, \phi_{0}\right)$, where $r_{0}$ in $\left(\mathbb{R}_{+} /\{0\}\right)^{n}$ is the weight, $d_{0}$ in $\mathbb{R}_{+}$the degree and $\phi_{0}: \mathbb{R}^{n} \rightarrow \mathbb{R}$ the approximating function, respectively, if $\phi_{0}$ is continuous and not identically zero and, for each compact set $C$ in $\mathbb{R}^{n} \backslash\{0\}$ and each $\varepsilon>0$, there exists $\lambda^{*}$ such that we have :

$$
\max _{x \in C}\left|\frac{\phi\left(\lambda^{r_{0}} \diamond x\right)}{\lambda^{d_{0}}}-\phi_{0}(x)\right| \leq \varepsilon \quad \forall \lambda \in\left(0, \lambda^{*}\right] .
$$

- A vector field $f=\sum_{i=1}^{n} f_{i} \frac{\partial}{\partial x_{i}}$ is said homogeneous in the 0-limit with associated triple $\left(r_{0}, d_{0}, f_{0}\right)$, where $f_{0}=\sum_{i=1}^{n} f_{0, i} \frac{\partial}{\partial x_{i}}$, if, for each $i$ in $\{1, \ldots, n\}$, the function $f_{i}$ is homogeneous in the 0 -limit with associated triple $\left(r_{0}, d_{0}+r_{0, i}, f_{0, i}\right)^{7}$.

Definition 2 (Homogeneity in the $\infty$-limit):

- A continuous function $\phi: \mathbb{R}^{n} \rightarrow \mathbb{R}$ is said homogeneous in the $\infty$-limit with associated triple $\left(r_{\infty}, d_{\infty}, \phi_{\infty}\right)$ where $r_{\infty}$ in $\left(\mathbb{R}_{+} /\{0\}\right)^{n}$ is the weight, $d_{\infty}$ in $\mathbb{R}_{+}$the degree and $\phi_{\infty}: \mathbb{R}^{n} \rightarrow \mathbb{R}$ the approximating function, respectively, if $\phi_{\infty}$ is continuous and not identically zero and, for each compact set $C$ in $\mathbb{R}^{n} \backslash\{0\}$ and each $\varepsilon>0$, there exists $\lambda^{*}$ such that we have :

$$
\max _{x \in C}\left|\frac{\phi\left(\lambda^{r_{\infty}} \diamond x\right)}{\lambda^{d_{\infty}}}-\phi_{\infty}(x)\right| \leq \varepsilon \quad \forall \lambda \in\left[\lambda^{*},+\infty\right) .
$$

- A vector field $f=\sum_{i=1}^{n} f_{i} \frac{\partial}{\partial x_{i}}$ is said homogeneous in the $\infty$-limit with associated triple $\left(r_{\infty}, d_{\infty}, f_{\infty}\right)$, with $f_{\infty}=\sum_{i=1}^{n} f_{\infty, i} \frac{\partial}{\partial x_{i}}$, if, for each $i$ in $\{1, \ldots, n\}$, the function $f_{i}$ is homogeneous in the $\infty$-limit with associated triple $\left(r_{\infty}, d_{\infty}+r_{\infty, i}, f_{\infty, i}\right)$.

Definition 3 (Homogeneity in the bi-limit):

A continuous function $\phi: \mathbb{R}^{n} \rightarrow \mathbb{R}$ (or a vector field $f$ ) is said homogeneous in the bi-limit if it is homogeneous in the 0 -limit and homogeneous in the $\infty$-limit.

Claim : Let $\eta$ and $\gamma$ be two continuous homogeneous in the bi-limit functions with weights $r_{0}, r_{\infty}$, degrees $d_{\eta, 0}, d_{\eta, \infty}$ and $d_{\gamma, 0}, d_{\gamma, \infty}$, and continuous approximating functions $\eta_{0}$, $\eta_{\infty}, \gamma_{0}, \gamma_{\infty}$

A.1. The function $x \mapsto \eta(x) \gamma(x)$ is homogeneous in the bilimit with associated triples $\left(r_{0}, d_{\eta, 0}+d_{\gamma, 0}, \eta_{0} \gamma_{0}\right)$ and $\left(r_{\infty}, d_{\eta, \infty}+d_{\gamma, \infty}, \eta_{\infty} \gamma_{\infty}\right)$.

A.2. If the degrees satisfy $d_{\eta, 0} \geq d_{\gamma, 0}$ and $d_{\eta, \infty} \leq d_{\gamma, \infty}$, and $\gamma(x) \geq 0$, and for $x \neq 0$

$$
\begin{aligned}
& \gamma(x)=0 \quad \Rightarrow \quad \eta(x)<0, \\
& \gamma_{0}(x)=0 \quad \Rightarrow \quad \eta_{0}(x)<0, \\
& \gamma_{\infty}(x)=0 \quad \Rightarrow \quad \eta_{\infty}(x)<0,
\end{aligned}
$$

then there exists a real number $k^{*}$ such that, for all $k \geq k^{*}$, and for all non zero $x$ in $\mathbb{R}^{n}$ :

$$
\eta(x)<k \gamma(x), \eta_{0}(x)<k \gamma_{0}(x), \eta_{\infty}(x)<k \gamma_{\infty}(x) .
$$

A.3. If the degrees satisfy $d_{\eta, 0} \geq d_{\gamma, 0}$ and $d_{\eta, \infty} \leq d_{\gamma, \infty}$ and the functions $\gamma, \gamma_{0}$ and $\gamma_{\infty}$ are positive definite then there exists a positive real number $c$ satisfying $\eta(x) \leq c \gamma(x)$ for all $x$ in $\mathbb{R}^{n}$.

\footnotetext{
${ }^{7}$ In the case of a vector field the degree $d_{0}$ can be negative as long as $d_{0}+r_{0, i} \geq 0$, for all $1 \leq i \leq n$.
} 